\title{
Medical Care after Cat Bites: A Typical Case and Review
}

\author{
Jarmo Oksi'1,2*, U. Ahlmén-Laiho ${ }^{3}$, M. Laine ${ }^{4}$ \\ ${ }^{1}$ Department of Infectious Diseases, Division of Medicine, Turku University Hospital, Turku, Finland \\ ${ }^{2}$ Faculty of Medicine, University of Turku, Turku, Finland \\ ${ }^{3}$ Department of Anesthesiology, Loimaa Regional Hospital, Loimaa, Finland \\ ${ }^{4}$ Department of Surgery, Loimaa Regional Hospital, Loimaa, Finland \\ Email: ${ }^{*}$ jarmo.oksi@utu.fi
}

Received 18 January 2014; revised 17 February 2014; accepted 16 March 2014

Copyright (C) 2014 by authors and Scientific Research Publishing Inc.

This work is licensed under the Creative Commons Attribution International License (CC BY).

http://creativecommons.org/licenses/by/4.0/

(c) (i) Open Access

\begin{abstract}
Pet cat bites are common-the most typical patient being a child, and the most typical locality hands and face. The normal flora of a pet cat's mouth houses very potent pathogens. Therapeutic measures include wound cleansing, prophylaxis against tetanus and possibly rabies. Antibiotic prophylaxis should be administered in the cases of all but the mildest superficial wounds especially if the wound is in the hand or face. We present a review on the medical care of cat bite wounds and a typical case of a patient who developed rapid symptoms and signs of infection after a pet cat bite and nearly required hospitalization. The clinical picture was consistent with a Pasteurella infection.
\end{abstract}

\section{Keywords}

Cat Bite; Animal Bite; Pasteurella; Antibiotic Prophylaxis

\section{Introduction}

Up to $90 \%$ of animal bites are caused by dogs and cats [1]. The most typical victim of a cat bite is a child [2]. Parents however are not well prepared to pet-associated bite hazards [3]. Cat bites account for up to $15 \%$ of all animal bites and usually affect the hands, arms and face [4]. Not all patients seek medical attention although up to $50 \%-80 \%$ of cat bites can get infected [5]-[7]. Potential complications may be serious including death [8]. We present a typical case of a patient who developed rapid symptoms and signs of infection after a pet cat bite and nearly required hospitalization. The clinical picture was consistent with a Pasteurella infection. General practitioners need to provide knowledge about prompt treatment to prevent or minimize infective complications

\footnotetext{
${ }^{*}$ Corresponding author.
} 
after cat or dog bites. We therefore gathered earlier and recently published literature for a review on cat bite wound infections. Therapeutic measures include wound cleansing, prophylaxis against tetanus and possibly rabies, and antibiotic prophylaxis especially in the case of cat bite wound in the hand or face. In cases that are not responding well to antibiotics surgical treatment should be considered.

\section{A Typical Case}

The patient was a 59-year-old immunocompetent female. She was bitten on her left thenar by a pet cat, and presented with two small puncture wounds. The patient administered satisfactory first aid cleansing and dressing of the wounds. Within four hours the hand became swollen, painful and warm. Medical attention was sought and oral amoxicillin-clavulanic acid 875/125 mg twice daily was instituted. In addition, a tetanus booster shot was given. On the following day her temperature was mildly elevated and the lymph nodes in her left armpit were swollen. On the second day after the bite general malaise and mild fever had subsided but a red line indicating lymphangitis was developing in the upper limb. The limb was observed carefully, and since the patient was feeling better, oral antibiotics and local treatment were continued. Signs of lymphangitis ameloriated in 3 - 4 days after which the patient was feeling good. The puncture wounds produced pus from day 2 to approximately day 4 . The patients required a sick leave of one week, and the antibiotics were continued for 10 days altogether.

\section{Discussion and Review of Literature}

The fact that cat bite wounds get infected more frequently (up to $50 \%-80 \%$ of cases) than do dog bite wounds (no more than $20 \%$ of cases) is probably related to sharper teeth of cats leading to deeper inoculation of bacteria and subsequent soft tissue abscesses or septic arthritis [4] [5] [9]. Criteria for animal bite wound infections were described by Talan et al. The major criteria are fever, abscess and lymphangitis. The minor criteria are erythema that extends more than $3 \mathrm{~cm}$ from the edge of the wound, wound site tenderness, swelling, purulent discharge, and white cell count over 12,000 per cubic ml of blood. A wound is considered to be infected if it meets one of the three major criteria or four of the five minor criteria [4]. Criteria for the need of hospitalization were described by Goldstein: severe cellulitis, systemic manifestations of infection (e.g. fever, vomiting, confusion), evidence of strong clinical suspicion of bone, joint, tendon or nerve involvement, rapid spread of infection within 24 - 48 hours, absence of response to oral outpatient therapy, and under certain circumstances patients regarded as unreliable or incompetent [10]. In a multicentre prospective study of 57 clinically infected human cat bite wounds, the most frequent type of infection was a non-purulent wound with cellulitis, lymphangitis, or both (42\%) followed by a purulent wound without (39\%) or with an abscess (19\%) [4]. The median time to the appearance of the first symptoms of infection are significantly shorter (often emerging in 3 hours) after a cat bite as compared to a dog bite [4] [11]. In a Swedish study of cat bite infections complications such as tenosynovitis, arthritis, cellulitis, abscesses and septicaemia occurred in $18 \%$ of patients [11]. Potential complications also include osteomyelitis-most commonly involving a finger or hand, septic shock or meningitis, endocarditis or distant abscesses as foci following septicaemia [4] [12]. Patients with a compromised immune system, including very young or elderly patients, are at greater risk in developing serious complications [12]. Prophylaxis against tetanus is recommended if the cat is allowed to roam outside, and against rabies if there raises a suspicion of a rabid cat for example via contact with bats that are the primary vectors of rabies.

Animal bite wound infections frequently are polymicrobial—up to $56 \%$ of infections are caused by a mix of aerobic and anaerobic bacteria [4] [13]. As many of the anaerobes are betalactamase producers, the combination of amoxicillin and clavulanic acid has been recommended as empirical treatment [4]. Culprit pathogens commonly belong to the normal flora of cat mouths. Pasteurella species are the most common and found in up to $75 \%$ or cat bite wounds where the subspecies P. multocida prevails over P. canis which in turn is the most prevalent in the case of dog bite wounds [4]. Pasteurellas are small gramnegative cocci. Typical symptoms of Pasteurella infections include rapidly (in less than 24 hours after the bite) developing pus formation along with quick classical symptoms and signs of local inflammation. Westling et al. found that the predominant symptoms in 78 patients with infection after a cat bite were erythema, pain and oedema, which often emerged as early as 3 hours after the bite. Pasteurella was cultured in the wound in 70\% of patients [11].

Local first aid of the wound by washing with clean water is of utmost importance. An open or jagged wound should be rinsed with saline using a large bore needle and syringe, although cat bites tend to have a small entry of wounds making this kind of cleansing impractical. Suturation of bite wounds is generally not recommended. 
In aesthetically critical areas such as the face, can it be cosmetically prudent sometimes to close the bite wound despite the fact that it increases the risk of deep infection.

Our patient was treated with antibiotics only after signs and symptoms of infection. However, it should be stressed that a proper strategy would have been to treat her with a 5-day course of prophylactic amoxicillin-clavulanic acid immediately after the bite. In fact, it is logical that prophylactic antibiotics improve the outcome after cat bites when majority of deeper puncture wounds lead to infection without it. However, patients might not seek medical attention until symptoms are already evident.

Antibiotic treatment of cat bites is recommended in all but the mildest surface injuries. Although majority of Pasteurellas are sensitive to amoxicillin is amoxicillin-clavulanic acid the preferred choice because of the frequency of mixed infections including anaerobic bacteria. The use of prophylactic antibiotics in mammalian bites was studied in a Cochrane review in 2001 and in an update in 2008 [14]. The main result was that prophylactic antibiotics were associated with a statistically significant reduction in the rate of infection in hand bites (OR $0.10,95 \%$ CI $0.01-0.86$; NNT $=4,95 \%$ CI 2 - 50). However, there was no evidence that the use of prophylactic antibiotics were effective when the wound was elsewhere in the body. There was only one study of cat bites that met the criteria for the review and investigated the benefit of using prophylactic antibiotics (and a few dog bite studies). It is important to notice that amoxicillin-clavulanic acid was not used as a prophylaxis in any of these prospective studies [15]. In a study over a 10-year period, hospitalization due to Pasteurella-induced animal biteassociated wound infection seemed to be related to the prescription of suboptimal oral antibiotic therapy at a preceding medical consultation [16]. Bite wounds in hand, on the face or genitalia, carry a large risk of infection and should be treated promptly regardless of whether symptoms of infection are present. The wound must be observed carefully and signs of abscesses or local unsatisfactory result of antibiotic treatment should be an indication to a surgical consultation or a change to intravenous treatment, usually with cefuroxime.

Lymphangitis is not always a symptom of progressing bacterial infection-it may also be an inflammatory reaction. In our case it likely was due to bacterial infiltration, but the overall well-being of the patient allowed a decision to continue with oral antibiotic treatment. Progressive lymphangitis that is suspected to be the result of rising bacterial infection generally indicates the need for hospitalization and intravenous treatment. In the absence of clearance of local signs a suspicion of abscess formation raises and surgical treatment should be considered.

\section{Conclusion}

We conclude that the normal flora of a pet cat's mouth houses very potent pathogens. Antibiotics should be administered in all but the mildest injuries regardless of whether there already are symptoms of infection. Our patient nearly required hospitalization after getting two very small puncture wounds on her left thenar.

\section{Conflicts of Interest}

J.O.: Lectures, congress travel, consultations (Abbott/AbbVie, Astellas, Baxter, Bristol-Myers Squibb, Gilead Sciences Finland, GlaxoSmithKline, MSD Finland, Pfizer, Ratiopharm, Tibotec/Janssen). Others: no conflicts of interest.

\section{References}

[1] Steele, M.T., Ma, O.J., Nakase, J., Moran, G.J., Mower, W.R., Ong, S., et al. Emergency ID NET Study Group (2007) Epidemiology of Animal Exposures Presenting to Emergency Departments. Academic Emergency Medicine, 14, 398403. http://dx.doi.org/10.1111/j.1553-2712.2007.tb01797.x

[2] Mitnovetski, S. and Kimble, F. (2004) Cat Bites of the Hand. ANZ Journal of Surgery, 74, 859-862. http://dx.doi.org/10.1111/j.1445-1433.2004.03189.x

[3] Villar, R.G., Connick, M., Barton, L.L., Meaney, F.J. and Davis, M.F. (1998) Parent and Pediatrician Knowledge, Attitudes, and Practices Regarding Pet-Associated Hazards. Archives of Pediatrics \& Adolescent Medicine, 152, 10351037. http://dx.doi.org/10.1001/archpedi.152.10.1035

[4] Talan, D.A., Citron, D.M., Abrahamian, F.M., Moran, G.J. and Goldstein, E.J.C. (1999) Bacteriologic Analysis on Infected Dog and Cat Bites. New England Journal of Medicine, 340, 85-92. http://dx.doi.org/10.1056/NEJM199901143400202

[5] Smith, P.F., Meadowcroft, A.M. and May, D.B. (2000) Treating Mammalian Bite Wounds. Journal of Clinical Phar- 
macy and Therapeutics, 25, 85-99. http://dx.doi.org/10.1046/j.1365-2710.2000.00274.x

[6] Dire, D.J. (1991) Cat Bite Wounds: Risk Factors for Infection. Annals of Emergency Medicine, 20, 973-979. http://dx.doi.org/10.1016/S0196-0644(05)82975-0

[7] Love, D.N. (2000) Bacteriological Warfare amongst Cats: What Have We Learned about Cat Bite Infections? Veterinary Microbiology, 74, 179-193. http://dx.doi.org/10.1016/S0378-1135(00)00186-3

[8] Normann, B., Niléhn, B., Rajs, J. and Karlberg, B. (1971) A Fatal Human Case of Pasteurella multocida Septicaemia after Cat Bite. Scandinavian Journal of Infectious Diseases, 3, 251-254.

[9] Esposito, S., Picciolli, I., Semino, M. and Principi, N. (2013) Dog and Cat Bite-Associated Infections in Children. European Journal of Clinical Microbiology \& Infectious Diseases, 32, 971-976. http://dx.doi.org/10.1007/s10096-013-1840-X

[10] Goldstein, E.J. (1992) Bite Wounds and Infection. Clinical Infectious Diseases, 14, 633-638. http://dx.doi.org/10.1093/clinids/14.3.633

[11] Westling, K., Farra, A., Cars, B., Ekblom, A.G., Sandstedt, K., Settergren, B., et al. (2006) Cat Bite Wound Infections: A Prospective Clinical and Microbiological Study at Three Emergency Wards in Stockholm, Sweden. Journal of Infection, 53, 403-407. http://dx.doi.org/10.1016/j.jinf.2006.01.001

[12] Weber, D.J., Wolfson, J.S., Swartz, M.N. and Hooper, D.C. (1984) Pasteurella multocida Infections. Report of 34 Cases and Review of the Literature. Medicine (Baltimore), 63, 133-154.

[13] Thomas, N. and Brook, I. (2011) Animal Bite Associated Infections: Microbiology and Treatment. Expert Review of Anti-Infective Therapy, 9, 215-226. http://dx.doi.org/10.1586/eri.10.162

[14] Medeiros, I. and Saconato, H. (2001) Antibiotic Prophylaxis for Mammalian Bites. Cochrane Database Systematic Reviews, 2, Article ID: CD001738.

[15] Henton, J. and Jain, A. (2012) Cochrane Corner: Antibiotic Prophylaxis for Mammalian Bites (Intervention Review). Journal of Hand Surgery, 37, 804-806. http://dx.doi.org/10.1177/1753193412452204

[16] Holm, M. and Tärnvik, A. (2000) Hospitalization Due to Pasteurella multocida-Infected Animal Bite Wounds: Correlation with Inadequate Primary Antibiotic Medication. Scandinavian Journal of Infectious Diseases, 32, 181-183. http://dx.doi.org/10.1080/003655400750045303 